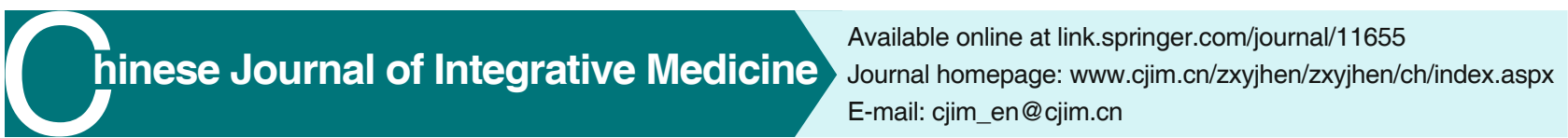

Original Article

\title{
Seabuckthorn Berries Extract Attenuates Pulmonary Vascular Hyperpermeability in Lipopolysaccharide-Induced Acute Lung Injury in Mice*
}

\author{
DU Lei-lei ${ }^{1}$, LIU Ying ${ }^{2}$, WAN Li ${ }^{3}$, CHEN Chu ${ }^{4}$, and FAN Gang ${ }^{1}$
}

\begin{abstract}
Objective: To investigate the effect of seabuckthorn berries extract (SBE) on pulmonary vascular hyperpermeability in the mice model of acute lung injury (ALI) induced by lipopolysaccharide (LPS). Methods: Sixty Kunming mice were allocated into 6 groups by a random number table, including control, LPS, dexamethasone (Dex, $1 \mathrm{mg} / \mathrm{kg}$ ), and 120, 240 and $480 \mathrm{mg} / \mathrm{kg}$ SBE groups, 10 mice in each group. Except the control group, mice were pre-treated with Dex and SBE, respectively, for 7 days before LPS was intraperitoneally injected to induce ALI. Pulmonary vascular hyperpermeability was evaluated by histopathologic observation and transvascular leakage determination. Tumor necrosis factor alpha (TNF- $\alpha$ ) and interleukin-6 (IL-6) levels in serum were measured using enzyme-linked immunosorbent assay. The expression of nuclear factorkappa B (NF- $\kappa$ B) p65 in lung cells was determined by immunofluorescence analysis. The contents of cytoplasmic inhibitor of nuclear factor- $\mathrm{K} B$ kinase (IKK) and nuclear p65, as well as downstream proteins of E-selectin (CD62E) and intercellular adhesion molecule-1 (ICAM-1), were determined using Western blot analysis. Results: Histopathological observation confirmed SBE treatment alleviated morphological lesion induced by LPS. Compared with the LPS group, $480 \mathrm{mg} / \mathrm{kg}$ SBE significantly decreased the water content of lung, Evans blue accumulation in lung tissue, and protein concentration and neutrophils count in bronchoalveolar lavage fluid $(P<0.01)$; moreover, $480 \mathrm{mg} / \mathrm{kg}$ SBE significantly suppressed release of TNF- $\alpha$ and IL-6, and down-regulated expressions of IKK, nuclear p65, ICAM-1 and CD62E $(P<0.01)$. Conclusion: SBE maintained alveolar-capillary barrier integrity under endotoxin challenge in mice by suppressing the key factors in the pathogenesis of ALI.
\end{abstract}

KEYWORDS seabuckthorn berries extract, pulmonary vascular hyperpermeability, acute lung injury, nuclear factor-kappa B, neutrophil

Acute lung injury (ALI) is a clinical syndrome characterized by progressive hypoxemia and respiratory distress, caused by diverse endogenous and exogenous factors that injure lung directly or indirectly. ALI is the source of substantial morbidity and mortality in both adult ${ }^{(1,2)}$ and pediatric ${ }^{(3,4)}$ populations and is a major contributor to intensive care unit costs. ${ }^{(5)}$ Sepsis, induced by severe infection of bacteria, virus or fungus, is one of the leading etiologies of ALI. ${ }^{(6)}$ It may cause sequencial functional disorders to multiple organs, among which $\mathrm{ALI}$ occurs at the earliest stage with the highest morbidity. ${ }^{(7,8)}$

Long-term clinical observations and experiments have demonstrated that $A L I$ is the distinct manifestation of systemic inflammatory response syndrome in lung. Severe inflammatory response and protein-rich edema fluids induced by vascular endothelial injury are presented through the entire process. ${ }^{(9)}$ Under pathogen stress, monocyte/ macrophage system is stimulated through nuclear factor-kappa B (NF- $\kappa$ B) signal pathway to release pro-inflammatory cytokines such as tumor necrosis factor alpha (TNF- $\alpha$ ) and interleukin-6 (IL-6), which in

CThe Chinese Journal of Integrated Traditional and Western Medicine Press and Springer-Verlag GmbH Germany, part of Springer Nature 2021

*Supported by the National Natural Science Foundation of China (No. 81503361)

1. School of Ethnic Medicine, Chengdu University of Traditional Chinese Medicine, Chengdu (611137), China; 2. School of Basic Medicine, Chengdu University, Chengdu (610106), China; 3. School of Pharmacy, Chengdu University of Traditional Chinese Medicine, Chengdu (611137), China; 4. Sichuan Provincial Key Laboratory of Quality and Innovation Research of Chinese Materia Medica, Sichuan Academy of Chinese Medicine Sciences, Chengdu (610041), China

Correspondence to: Prof. FAN Gang, E-mail: fangang1111@163. com

DOI: https://doi.org/10.1007/s11655-021-3346-1 
turn mediate adhesion and combination of neutrophils to endothelial cells in pulmonary microvessels by regulation of adhesion molecules such as intercellular adhesion molecule-1 (ICAM-1) and E-selectin (CD62E). ${ }^{(10,11)}$ Activation of neutrophils results in the release of mediators (e.g., oxidants and proteases) that increase vascular permeability by disrupting interendothelial junctions, thereby intravascular fluid and macromolecules permeate into alveoli and cause edema formation in pulmonary tissue.

Pharmacological therapies are still sparse for $A L I$, and supportive treatment of low tidal volume ventilation is the main strategy in clinical practice. ${ }^{(12)}$ However, mechanical tension generated by ventilation itself may introduce inflammatory response and cause further injury. ${ }^{(13)}$ Therefore, searching for alternative therapeutic strategy is of substantial interests.

Seabuckthorn (Hippophae rhamnoides L., Elaegnaceae) berries have been used to treat lung diseases in traditional Tibetan medicine for a long history. Modern research revealed that the main active constituents of seabuckthorn berries are flavanoids, which present potent anti-oxidative and anti-inflammatory capacities. ${ }^{(14)}$ In the current work, we investigated the effect of seabuckthorn berries on lipopolysaccharide (LPS)-induced ALI in mice and explored the possible mechanism.

\section{METHODS}

\section{Chemicals and Reagents}

Isorhamnetin (lot No. MUST-12112001) and quercetin (lot No. MUST-13072505) reference standards were obtained from Must Bio-technology Company (Chengdu, China). Kaempferol (lot No. 110861-201209) reference standard was obtained from Chinese National Institutes of Food and Drug Control (Beijing, China). Dexamethasone acetate tablet (Dex, lot No. 200527205) was purchased from Cisen Pharmaceutical Co, Ltd. (Jining, China). Evans blue dye (lot No. E2129) and LPS (O55:B5, lot No. $110 M 4095)$ was purchased from Sigma (MO, USA). Bicinchoninic acid (BCA, lot No. 20201024), TNF- $\alpha$ (lot No. 20201018) and IL-6 (lot No. 20200917) enzyme-linked immunosorbent assay (ELISA) kits were purchased from Jiancheng Bioengineering Institute (Nanjing, China). Rabbit anti-NF- к B (p65) (lot No. 23303), rabbit anti-inhibitor of nuclear factor- $\kappa$ B kinase (IKK, lot No. 23404), mouse anti-
ICAM-1 (lot No. 23302), rabbit anti-CD62E (lot No. 23401) antibodies, and 2-(4-amidinophenyl)-6indolecarbamidine dihydrochloride (DAPI, lot No. 23303) were purchased from Abcam (MA, USA). The nuclear extract kit (lot No. 40010) was purchased from Active Motif (CA, USA). The polyvinylidene fluoride (PVDF, lot No. ISEQ00010) membrane was purchased from Millipore (MA, USA).

\section{Plant Material and Extract Preparation}

Well-ripened seabuckthorn berries were collected from natural growth site of hilly region in eastern margin of Tibetan Plateau (Ma'erkang, Sichuan province, China) and identified by CHEN Chu as $H$. rhamnoides L., Elaegnaceae. Voucher specimens (No. MZC-SJ-20180924-00 03) of the plant material are preserved in the herbarium of Chengdu University of Traditional Chinese Medicine after botanical identification.

The berries were refluxed with deionized water and the supernatant was concentrated under vacuum to achieve seabuckthorn berries extract (SBE), with a yield of $9.22 \%$. Contents determination of isorhamnetin, quercetin and kaempferol were performed on an Acquity ultra-performance liquid chromatography (UPLC, Waters, USA) and a Waters BEH C18 column $(50 \mathrm{~mm} \times 2.1 \mathrm{~mm}, 1.7 \mu \mathrm{m})$ was used. The mobile phase consisted of acetonitrile- $0.2 \%$ aqueous acetic acid (28:72). The flow rate was $0.4 \mathrm{~mL} / \mathrm{min}$ and column temperature was maintained at $30{ }^{\circ} \mathrm{C}$. The detection wavelength was set at $360 \mathrm{~nm}$. Samples of $2 \mu \mathrm{L}$ were injected.

\section{Animals, Grouping and Treatment}

Sixty male Kunming mice of specified pathogen free grade (6-8 weeks old, 18-22 g) were obtained from Chengdu Dossy Experimental Animals Co., Ltd., China [certificate No. SCXK (Chuan) 2020-030]. Mice were kept in a controlled environment $\left(24 \pm 5{ }^{\circ} \mathrm{C}\right.$, $55 \%$ humidity, $12 \mathrm{~h} / 12 \mathrm{~h}$ light/dark cycle), ad libitum access to food and water. The experiment was approved by the Institute's Animal Ethical Committee (No. CDUTCM20200312) and confirmed to national guidelines on the use and care of laboratory animals.

After acclimatization for 2 days, mice were allocated into 6 groups by a random number table, including control, LPS, Dex (1 mg/kg), and 120 , 240 and $480 \mathrm{mg} / \mathrm{kg} \mathrm{SBE}$ groups, 10 mice in each 
group. Dex, LPS and SBE were dissolved in saline respectively to yield solutions of proper concentration. Mice in each group were received respective treatment (saline for control and LPS groups) once daily through intragastric route for 7 consecutive days. On Day 8, mice were administered with saline for the control group and LPS (O55:B5, $10 \mathrm{mg} / \mathrm{kg}$ ) for other groups by intraperitoneal injection. Ten hours later, mice were anesthetized by pentobarbital sodium and sacrificed by cervical dislocation.

\section{Gross and Histopathologic Observation}

After the animal was sacrificed, median sternotomy was performed to expose trachea and pleuroperitoneal cavity, so that lung was excised for visual inspection. Then the lung was fixed with an intratracheal instillation of $1 \mathrm{~mL}$ buffered formalin (10\%, pH 7.2). The lobe was further fixed in $10 \%$ neutral buffered formalin for $48 \mathrm{~h}$ at $4{ }^{\circ} \mathrm{C}$. The tissues were embedded in paraffin wax.

\section{Lung Water Content Determination}

After the animal was sacrificed, lungs were excised en bloc, blot dried and placed on pre-weighed glass plates. The wet weight of the tissue was registered immediately. Then the tissue was placed in an incubator at $80{ }^{\circ} \mathrm{C}$ for $72 \mathrm{~h}$ to obtain a constant weight. After dry weight of the tissue was recorded, water content of the tissue was calculated as wet weight/dry weight ratio (W/D).

\section{Transvascular Leakage Analysis}

In order to evaluate LPS-induced lung vascular leak, $1 \%$ Evans blue dye in saline $(10 \mathrm{~mL} / \mathrm{kg})$ was injected into the tail vein of mice $1 \mathrm{~h}$ before termination of the experiment. Measurement of Evans blue accumulation in the lung tissue was performed by spectrofluorimetric analysis of lung tissue lysates according to the protocol described previously. ${ }^{(15,16)}$

Protein content in bronchoalveolar lavage fluid (BALF) reflects macromolecule leakage through impaired endothelia barrier. To analyze BALF, animal's trachea was exposed and an intravenous infusion needle was inserted. The lungs were lavaged thrice with $0.5 \mathrm{~mL}$ of ice-cold phosphatebuffered saline. Returned lavage fluid was pooled for each animal and centrifuged at $800 \times g$ for $5 \mathrm{~min}$ at $4{ }^{\circ} \mathrm{C}$. The supernatants were harvested for total protein analysis using BCA protein assay kit and the sediments were collected for neutrophils count under light microscope on cytospin slides stained with Wright's solution.

\section{Inflammatory Cytokines Assay}

To evaluate inflammatory response, blood samples from the abdominal aorta of mice were collected before the animals were sacrificed, followed by incubation for $1 \mathrm{~h}$ under $37^{\circ} \mathrm{C}$ and centrifugation for $5 \mathrm{~min}$ at $1,500 \times \mathrm{g}$. Serum cytokines TNF- $\alpha$ and IL-6 levels were measured using ELISA kits according to the manufacturer's instructions.

\section{Immunofluorescent Analysis}

The expression of NF- $\kappa$ B p65 in lung cells was determined by immunofluorescence technique. Sections of lung tissue were deparaffinized and rehydrated through submersion in graded alcohols. Antigen retrieval was performed with $10 \mathrm{mmol} / \mathrm{L}$ citrate buffer $(\mathrm{pH} 6)$, for $5 \mathrm{~min}$ in a microwave oven. The sections were incubated with a primary antibody against NF- $\kappa$ B p65 (1:400), followed by detection with a fluorescein-conjugated secondary antibody (1:100). The nuclei were counterstained with DAPI. The fluorescent images were captured using appropriate filters in a Nikon inverted fluorescent microscope (Tokoyo, Japan), and integrated optical density (IOD) and percent of positive area (PPA) for photomicrographs were calculated using image processing software Image-Pro Plus 6.0 (Media Cybernetics, USA).

\section{Western Blot Analysis}

To evaluate the expression of proteins relative to NF- $\kappa$ B signal pathway, the contents of cytoplasmic IKK and nuclear p65, as well as downstream CD62E and ICAM-1, were determined using Western blot analysis. Lung tissue homogenate were centrifugation $\left(12,000 \times g, 10 \mathrm{~min}, 4{ }^{\circ} \mathrm{C}\right)$ and supernatants were aspirated. Biochemical fractionation of the cells was done using the nuclear extract kit according to the manufacturer's instructions. Proteins were loaded and transferred to a PVDF membrane. After being blocked, membranes were incubated overnight at $4{ }^{\circ} \mathrm{C}$ with a primary antibody, followed by incubation with secondary antibody for $1 \mathrm{~h}$ at room temperature. The membranes were placed into a gel imaging system (ChemiDoc XRS, Bio-Rad, USA) and then exposed. The intensity of blots was quantified using the Quantity One Analysis software (Bio-Rad). 


\section{Statistical Analysis}

All statistical analysis was performed with Prism 8 software (GraphPad Software, USA). Data were expressed as mean \pm standard deviation $(\bar{x} \pm s)$. Comparisons between groups were determined by one-way analysis of variance followed by Tukey's test. Results were considered statistically significant if $P$-values were $<0.05$.

\section{RESULTS}

Contents of Isorhamnetin, Quercetin and Kaempferol

Figure 1 shows UPLC chromatogram of reference standards, SBE and SBE hydrolysate. The contents of isorhamnetin, quercetin and kaempferol in SBE and SBE hydrolysate are listed in Appendix 1. The increased contents of 3 aglycones in SBE hydrolysate than those in SBE represented isorhamnetin, quercetin and kaempferol in form of various glycosides in SBE, which were hydrolyzed into aglycones by acid.
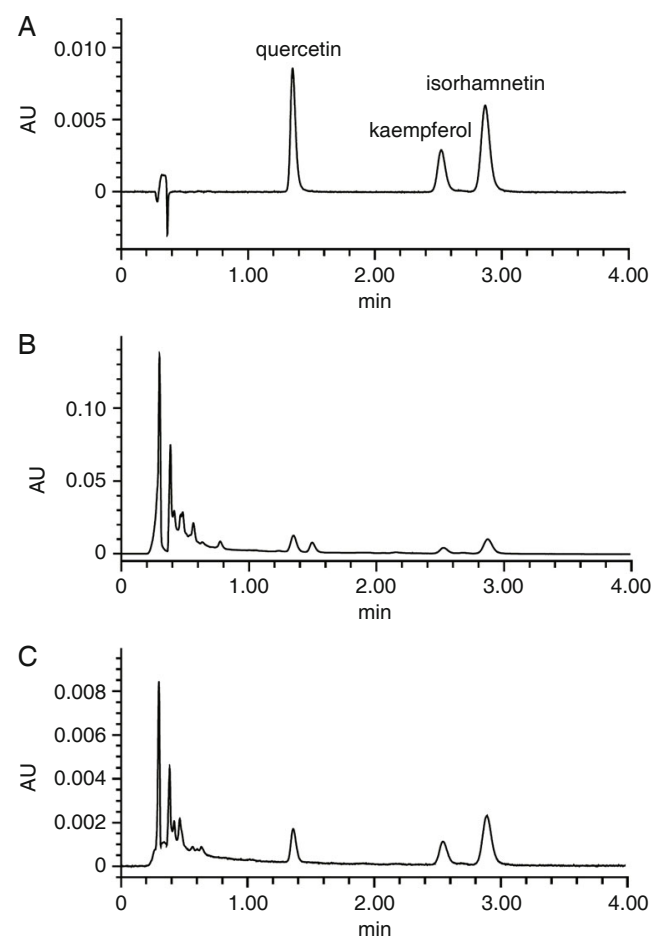

Figure 1. UPLC Chromatograms of Mixed Reference Standards (A), SBE (B) and SBE Hydrolysate (C)

\section{Morphological Changes upon LPS Challenge}

As shown in Figure 2, compared with healthy tissue of the control group, LPS stimulation caused obvious lesion in lungs of model mice, including foamy mucus in some tracheas, enlarged lobes, darker in color with scattered petechiae, and incisions exudation. Dex and SBE treatment provided protection to a certain degree, with alleviated edematous lesion and fewer petechiae observed.
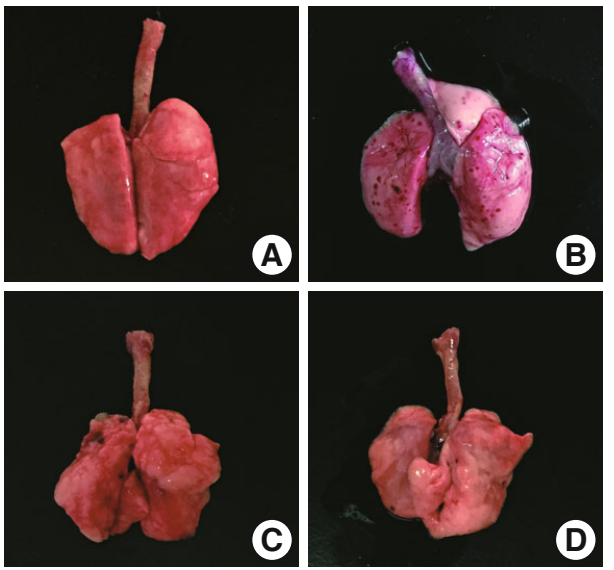

Figure 2. Effect of LPS on Lung Morphology $(n=10)$ Notes: A: control group; B: model group; C: Dex group; D: SBE $480 \mathrm{mg} / \mathrm{kg}$ group

\section{Effects of SBE on Lung Vascular Permeability}

Compared with the control group, the stimulation of LPS caused edema in lung tissue, reflected in a remarkable increase of W/D ratio $(P<0.01)$. However, pretreatment with Dex and SBE $480 \mathrm{mg} / \mathrm{kg}$ reversed the increase $(P<0.01$, Figure $3 A)$.

Evans blue accumulation in lung tissue through transvascular leakage was remarkable higher in the LPS group than that in the control group $(P<0.01)$, while SBE and Dex remarkably reserved the increase $(P<0.01$ or $P<0.05)$. Protein concentration and neutrophils count in BALF both sharply increased in the LPS group, compared with those in the control group $(P<0.01)$. Dex and SBE 480 and $240 \mathrm{mg} / \mathrm{kg}$ groups all showed significant decreases in protein concentration of BALF (all $P<0.01$ ), and Dex and 3 doses of SBE groups all showed obvious decreases in neutrophils count (all $P<0.01$, Figures 3B-3D).

\section{Effects of SBE on Inflammatory Cytokines}

The TNF- $\alpha$ and IL- 6 levels in serum were higher in the LPS group than those in the control group $(P<0.01)$. Compared with the LPS group, the TNF- $\alpha$ level was significantly decreased in the SBE $480 \mathrm{mg} / \mathrm{kg}$ and Dex groups, and IL-6 level was significantly decreased in all SBE and Dex groups (all $P<0.01$, Figure 4).

\section{Effects of SBE on NF- $\kappa$ B Activation}

The immunofluorescence images showed 

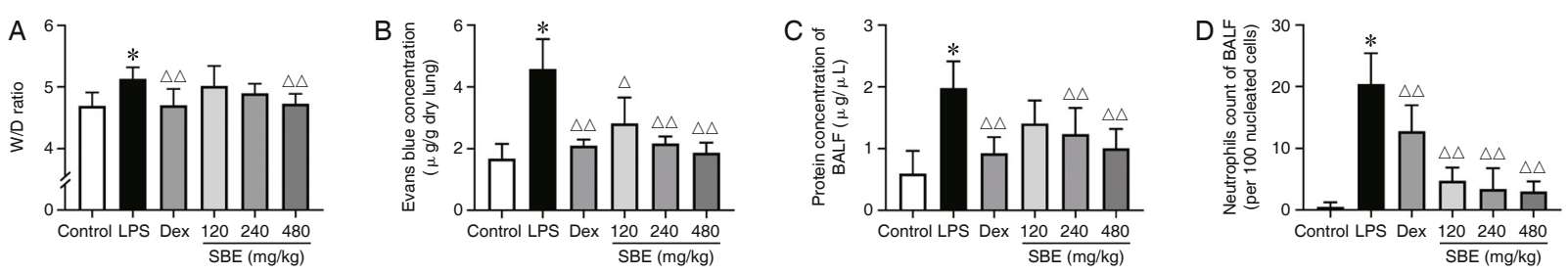

Figure 3. Effects of SBE on Lung Vascular Permeability $(\bar{x} \pm s, n=10)$

Notes: SBE: seabuckthorn berries extract; LPS: lipopolysaccharide; Dex: dexamethasone; BALF: bronchoalveolar lavage fluid; W/D: wet weight/dry weight ratio. ${ }^{*} P<0.01$ vs. control group; ${ }^{\Delta} P<0.05,{ }^{\Delta \Delta} P<0.01$ vs. LPS group
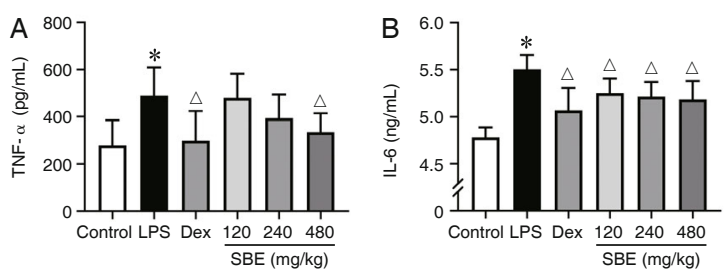

Figure 4. Effects of SBE on Cytokines TNF- $\alpha$ and IL-6 Levels $(\bar{x} \pm s, n=10)$

Notes: SBE: seabuckthorn berries extract; LPS: lipopolysaccharide; Dex: dexamethasone; TNF- $\alpha$ : tumor necrosis factor alpha; IL-6: interleukin-6. ${ }^{*} P<0.01$ vs. control group; ${ }^{\triangle} P<0.01$ vs. LPS group

enrichment of NF- $\kappa$ B p65 protein both in the nuclear and cytoplasmic fraction of lung cells in the LPS group, with green fluorescence for NF- $\kappa$ B p65 protein and blue fluorescence for nuclear. In the SBE $480 \mathrm{mg} / \mathrm{kg}$ group, fluorescence intensity appeared weaker than that of LPS group (Figure 5A). Furthermore, IOD and PPA assays both indicated SBE $480 \mathrm{mg} / \mathrm{kg}$ treatment significantly reversed the increased
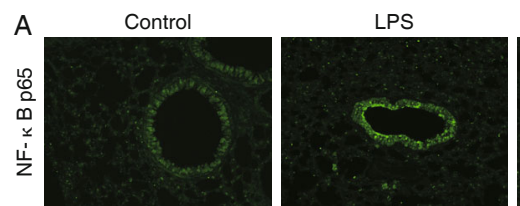

SBE $480 \mathrm{mg} / \mathrm{kg}$
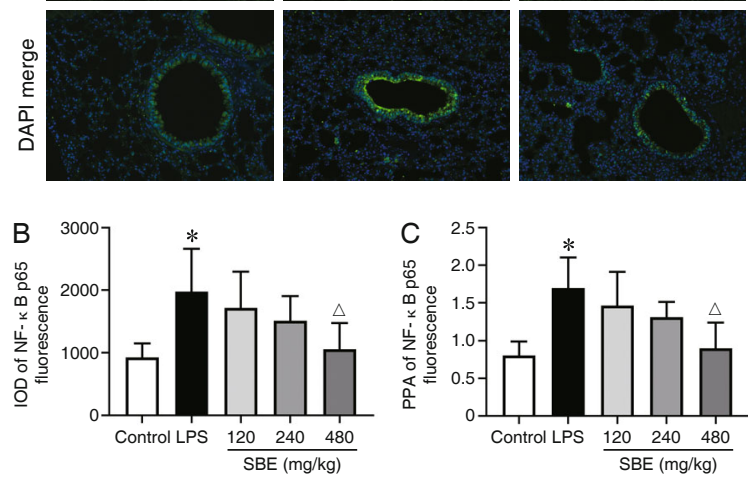

Figure 5. Effects of SBE on NF- $\kappa$ B p65 Immunofluorescence Images (A, $\times 400)$, IOD (B) and PPA (C) Analysis of Fluorescence Intensity $(\bar{x} \pm s, n=10)$

Notes: IOD: integrated optical density; PPA: percent of positive area; SBE: seabuckthorn berries extract; LPS: lipopolysaccharide; NF- $\kappa$ B: nuclear factor-kappa B. ${ }^{*} P<0.01$ vs. control group; ${ }^{\triangle} P<0.01$ vs. LPS group immunofluorescence intensity induced by LPS $(P<0.01$, Figures 5B and 5C).

The results of Western blot showed that low basal expression levels of cytoplasmic IKK and nuclear p65 increased significantly upon LPS stimulation $(P<0.01)$. By contrast, the expressions of IKK and nuclear p65 decreased notably in 3 doses of SBE groups $(P<0.01$, Figure 6$)$. Similarly, treatment of 480 and $240 \mathrm{mg} / \mathrm{kg}$ SBE reserved LPS-induced increased expressions of two downstream proteins of ICAM-1 and CD62E $(P<0.01$, Figure 7$)$.
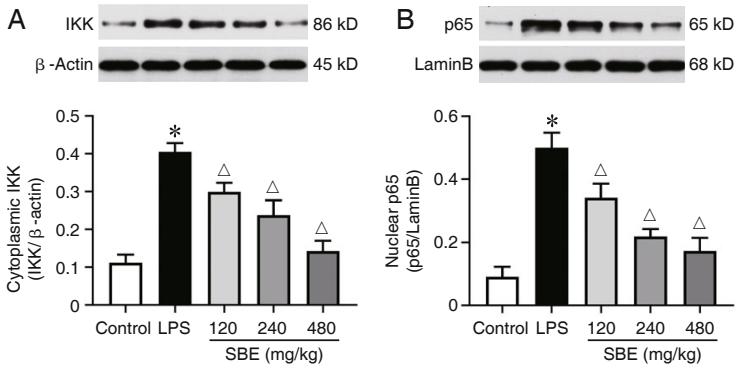

Figure 6. Effects of SBE on Expressions of

Cytoplasmic IKK and Nuclear p65 $(\bar{x} \pm s, n=6)$

Notes: SBE: seabuckthorn berries extract; LPS: lipopolysaccharide; IKK: inhibitor of nuclear factor- $\kappa$ B kinase. ${ }^{*} P<0.01$ vs. control group; ${ }^{\triangle} P<0.01$ vs. LPS group
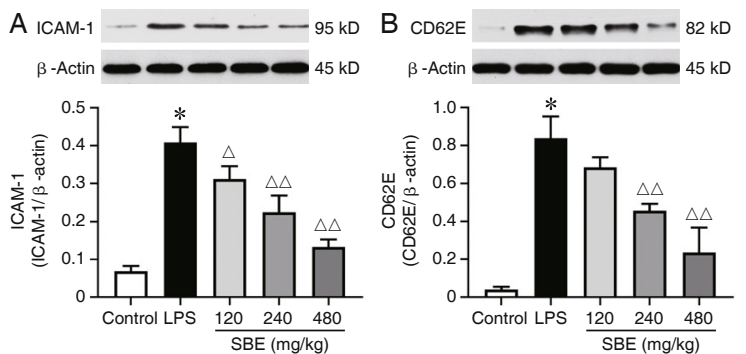

Figure 7. Effects of SBE on Protein Expressions of ICAM-1 and CD62E ( $\bar{x} \pm s, n=6)$

Notes: SBE: seabuckthorn berries extract: LPS: lipopolysaccharide; ICAM-1: intercellular adhesion molecule-1. ${ }^{*} P<0.01$ vs. control group; ${ }^{\triangle} P<0.05,{ }^{\triangle} P<0.01$ vs. LPS group

\section{DISCUSSION}

In the present study, we investigated the effect of SBE on ALI in mice, inspired by its therapeutic use 
in traditional Tibetan medicine for treating various pulmonary diseases and relieving hypoxic respiratory distress. Since sepsis is the most common clinical setting in which ALI develops and bacterial endotoxin is implicated as an important toxin precipitating lung injury, the widely-accepted sepsis-related lung injury model by LPS administration was used. Anatomical findings revealed severe pulmonary tissue injuries induced by LPS, including foamy mucus and scattered petechiae, were alleviated to some extent by SBE treatment.

Airway vascular endothelial injury is a major pathological feature of ALI. Endotoxin induces inflammatory response, with accumulation of inflammatory mediators in lung tissue, causing alveolar-capillary barrier lesion, which is associated with increased vascular permeability and accumulation of protein-rich interstitial and alveolar fluid. We determined lung water content by W/D ratio, which increased obviously in the LPS group, indicating pulmonary edema in model animals. Albumin leakage was determined by Evans blue assay. Intravenously administrated Evans blue binds to serum albumin with high affinity and serves as a probe to trace albumin leakage. ${ }^{(17)}$ Elevated level of Evans blue concentration in lung tissue of LPS group showed pulmonary vascular hyperpermeability. This is verified by increased total protein concentration in BALF of LPS group. These findings demonstrated the integrity of alveolar-capillary barrier was impaired in LPS-induced ALI mice, consistent with the other reports. ${ }^{(12,15,17)}$ By contrast, data in SBE groups showed alleviation of transvascular leakage, suggesting that SBE can protect alveolarcapillary barrier integrity upon endotoxin challenge.

Activation of neutrophils sequestered in pulmonary microvessels is an important factor in the pathogenesis of increased lung vascular permeability and tissue injury. ${ }^{(18)}$ We found in this experiment that the elevated count of neutrophils in BALF induced by LPS was reversed by SBE treatment, which is consistent with the endothelial permeability results.

Inflammation is associated with the pathological process of ALI. Pro-inflammatory cytokines TNF- $\alpha$ and IL- 6 have been strongly implicated in the pathogenesis of $A L I$ in human and animal models. ${ }^{(19)}$ Our results confirmed that SBE curtailed TNF- $\alpha$ and IL-6 release induced by LPS, showing significant antiinflammatory activity.
Since NF- к B signal pathway plays a key role in inflammatory response, we assumed it may be the effect target of SBE. In a quiescent state, NF- $\kappa$ B dimers are anchored in the cytoplasm by inhibitor of NF- $\kappa B(I \kappa B)$. When activated by signals, IKK degrades $I \kappa B$ through phosphorylation and ubiquitination, and NF- $\kappa \mathrm{B}$ is then freed to enter the nucleus where it can turn on the expression of relative genes. ${ }^{(20)}$ We investigated NF- $\kappa$ B expression in lung tissue by immunofluorescent analysis, as well as cytoplasmic IKK and nuclear NF- $\kappa$ B p65 expression by Western blot. The results demonstrated that SBE suppressed the expression of IKK and the nuclear translocation of NF- $\kappa$ B stimulated by LPS. The downstream proteins of NF- $\kappa B$ relative to neutrophil activation include ICAM-1 and CD62E, which mediate neutrophil interaction with endothelial cells. We also evaluated the expression levels of the two proteins and confirmed the similar suppression effect by SBE. These findings provide consistent evidences supporting the inhibition activity of SBE on NF- $\kappa$ B signal pathway.

The notable alveolar-capillary barrier protection and anti-inflammtory activity of SBE may attribute to multiple constituents. The main active ingredients include flavonoids, a kind of natural anti-oxidative and anti-inflammtory agents. Total flavonoids from seabuckthorn have been used in treating cardiovascular disease. Also, the high content of vitamins ( $\mathrm{B}, \mathrm{C}, \mathrm{E}$ and $\mathrm{K}$ ) makes seabuckthorn a popular nutritional supplement. Oil from seabukchtorn berries containing fatty acids and carotenoids facilitates the wound healing in burning and ulcer. ${ }^{(21)}$ Therefore, SBE may serve as a natural complex preparation to provide beneficial effect in ALI mice. The specific contribution for individual component merits further investigation.

In the present research, we demonstrated that SBE can protect alveolar-capillary barrier from hyperpermeability in LPS-induced ALI mice by suppressing the key factors in the pathogenesis of $A L I$, including the release of cytokines TNF- $\alpha$ and IL-6, the activation of NF- $\kappa$ B signal pathway, the expressions of ICAM-1 and CD62E, and the adhesion of neutrophil to endothelial cell. The effective constituents need further research and development, in order to provide a supplemental preventive and therapeutic strategy for ALI. 


\section{Conflict of Interest}

The authors declare that they have no conflicts of interest.

\section{Author Contributions}

Fan $\mathrm{G}$ and Chen $\mathrm{C}$ designed the research; Du LL, Liu $Y$, and Wan $L$ performed research; Chen $C$ and Liu $Y$ collected seabuckthorn samples and identified the specimen; Du LL and Fan $\mathrm{G}$ wrote the paper.

Electronic Supplementary Material: Supplementary material (Appendix 1) is available in the online version of this article at https://doi.org/10.1007/s11655-021-3346-1.

\section{REFERENCES}

1. Dushianthan A, Cusack R, Burgess VA, Grocott MP, Calder PC. Immunonutrition for acute respiratory distress syndrome (ARDS) in adults. Cochrane Database Syst Rev 2019;1:CD012041.

2. Chen YB, Liu Q, Xie H, Yin SM, Wu L, Yu XH, et al. Is Chinese medicine injection applicable for treating acute lung injury and acute respiratory distress syndrome? A systematic review and meta-analysis of randomized controlled trials. Chin J Integr Med 2020;26:857-866.

3. Randolph AG. Management of acute lung injury and acute respiratory distress syndrome in children. Crit Care Med 2009;37:2448-2454.

4. Rufener C, Kopstick A, Orwoll B. Pediatric ARDs: clinician recognition since the release of the pediatric acute lung injury consensus conference definition. Chest 2020;158:A1856.

5. Matthay MA, Zemans RL, Zimmerman GA, Arabi YM, Beitler JR, Mercat A, et al. Acute respiratory distress syndrome. Nat Rev Dis Primers 2019;5:18.

6. Johnson ER, Matthay MA. Acute lung injury: epidemiology, pathogenesis, and treatment. J Aerosol Med Pulm Drug Deliv 2010;23:243-252.

7. Matthay MA, Zimmerman GA, Esmon C, Bhattacharya J, Coller B, Doerschuk CM, et al. Future research directions in acute lung injury: summary of a National Heart, Lung, and Blood Institute working group. Am J Respir Crit Care Med 2003;167:1027-1035.

8. Martin GS, Mannino DM, Eaton S, Moss M. The epidemiology of sepsis in the United States from 1979 through 2000. N Engl J Med 2003;348:1546-1554.

9. Pierrakos C, Karanikolas M, Scolletta S. Acute respiratory distress syndrome: pathophysiology and therapeutic options. J Clin Med Res 2012;4:7-16.
10. Xu FB, Ji Q, Zhang J, Huang WY, Cao Z, Li YF. AICI3 inhibits LPS-induced NLRP3 inflammasome activation and IL-1beta production through suppressing NFkappaB signaling pathway in murine peritoneal macrophages. Chemosphere 2018;209:972-980.

11. Raham A, Fazal F. Hug tightly and say goodbye: role of endothelial ICAM-1 in leukocyte transmigration. Antioxid Redox Signal 2009;11:823-839.

12. Varisco BM. The pharmacology of acute lung injury in sepsis. Adv Pharmacol Sci 2011;2011:254619.

13. Fan E, Del SL, Goligher EC, Hodgson CL, Munshi L, Walkey AJ, et al. An official American Thoracic Society/European Society of Intensive Care Medicine/Society of Critical Care Medicine clinical practice guideline: mechanical ventilation in adult patients with acute respiratory distress syndrome. Am J Respir Crit Care Med 2017;195:1253-1263.

14. Bal LM, Meda V, Naik S, Satya S. Sea buckthorn berries: a potential source of valuable nutrients for nutraceuticals and cosmeceuticals. Food Res Int 2011;44:1718-1727.

15. Rojas M, Woods CR, Mora AL, Xu J, Brigham KL. Endotoxin-induced lung injury in mice: structural, functional, and biochemical responses. Am J Physiol Lung Cell Mol Physiol 2005;288:L333-L341.

16. Moitra J, Sammani S, Garcia JG. Re-evaluation of Evans blue dye as a marker of albumin clearance in murine models of acute lung injury. Transl Res 2007;150:253-265.

17. Birukova AA, Meng F. Prostacyclin post-treatment improves LPS-induced acute lung injury and endothelial barrier recovery via Rap1. Biochim Biophys Acta 2015;1852:778-791.

18. Kapur R, Kasetty G, Rebetz J, Egesten A, Semple JW. Osteopontin mediates murine transfusion-related acute lung injury via stimulation of pulmonary neutrophil accumulation. Blood 2019;134:74-84.

19. Li X, Ye C, Mulati M, Sun L, Qian F. Ellipticine blocks synergistic effects of IL-17A and TNF- $\alpha$ in epithelial cells and alleviates severe acute pancreatitis-associated acute lung injury. Biochem Pharmacol 2020;177:113992.

20. Xin WX, Li QL, Fang L, Zhong LK, Zheng XW, Huang P. Preventive effect and mechanism of ethyl acetate extract of Sceptridium ternatum in monocrotaline-induced pulmonary arterial hypertension. Chin J Integr Med 2020;26:205-211.

21. Singh IP, Ahmad F, Gore DD, Tikoo K, Bansal A, Jachak SM, et al. Therapeutic potential of seabuckthorn: a patent review (2000 - 2018). Expert Opin Ther Pat 2019;29:733-744.

(Accepted July 9, 2021; First Online December 7, 2021) Edited by YU Ming-zhu 